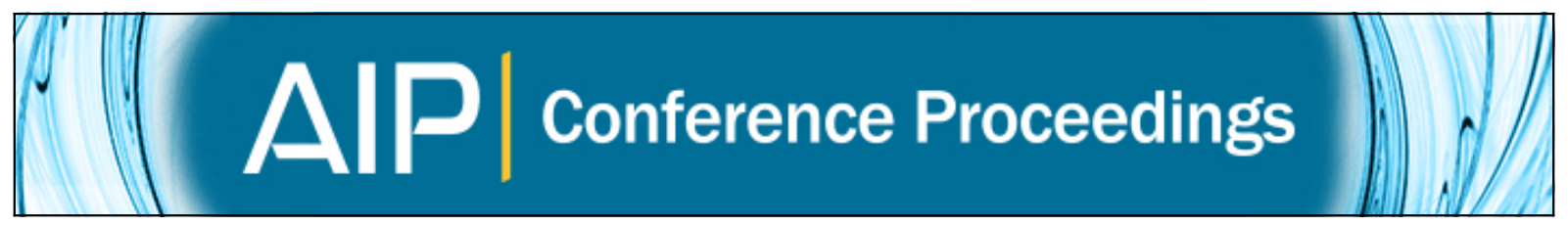

\title{
Perspectives for neutron and gamma spectroscopy in high power laser driven experiments at ELI-NP
}

F. Negoita, M. Gugiu, H. Petrascu, C. Petrone, D. Pietreanu, J. Fuchs, S. Chen, D. Higginson, L. Vassura, F. Hannachi, M. Tarisien, M. Versteegen, P. Antici, D. Balabanski, S. Balascuta, M. Cernaianu, I. Dancus, S. Gales , L. Neagu, C. Petcu, M. Risca, M. Toma, E. Turcu, and D. Ursescu

Citation: AIP Conference Proceedings 1645, 228 (2015); doi: 10.1063/1.4909579

View online: http://dx.doi.org/10.1063/1.4909579

View Table of Contents: http://scitation.aip.org/content/aip/proceeding/aipcp/1645?ver=pdfcov

Published by the AIP Publishing

\section{Articles you may be interested in}

Strong field physics and QED experiments with ELI-NP $2 \times 10 \mathrm{PW}$ laser beams

AIP Conf. Proc. 1645, 416 (2015); 10.1063/1.4909613

Positron production at extreme light infrastructure - nuclear physics (ELI-NP)

AIP Conf. Proc. 1645, 372 (2015); 10.1063/1.4909604

A conceptual design of an electron spectrometer for ELI-NP

AIP Conf. Proc. 1645, 296 (2015); 10.1063/1.4909589

Gamma beam system at ELI-NP

AIP Conf. Proc. 1645, 237 (2015); 10.1063/1.4909580

High power femtosecond lasers at ELI-NP

AIP Conf. Proc. 1645, 219 (2015); 10.1063/1.4909578 


\title{
Perspectives for neutron and gamma spectroscopy in high power laser driven experiments at ELI-NP
}

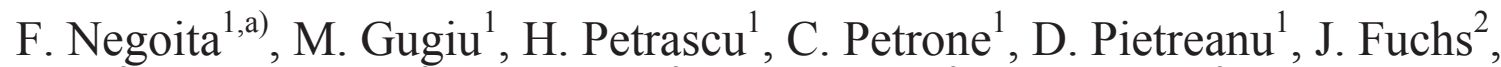 \\ S. Chen ${ }^{2}$, D. Higginson ${ }^{2}$, L. Vassura ${ }^{2}$, F. Hannachi ${ }^{3}$, M. Tarisien ${ }^{3}$, M. Versteegen ${ }^{3}$, \\ P. Antici ${ }^{2,4}$, D. Balabanski ${ }^{5}$, S. Balascuta ${ }^{5}$, M. Cernaianu, ${ }^{5}$ I. Dancus ${ }^{5}$, S. Gales ${ }^{5}$,

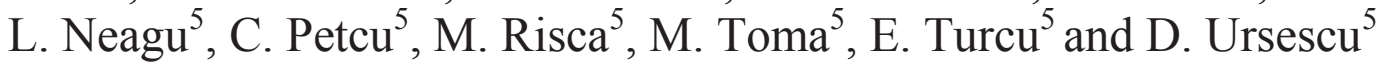 \\ ${ }^{1}$ IFIN-HH, Str. Reactorului nr. 30, 077125 Bucharest-Magurele, Romania \\ ${ }^{2}$ LULI, UMR 7605 CNRS-CEA-EcolePolytechnique-Universite Paris VI, 91128 Palaiseau, France \\ ${ }^{3}$ Universite Bordeaux 1, CENBG, CNRS-IN2P3, Route du solarium, 33175 Gradignan, France \\ ${ }^{4}$ Univ. Roma La Sapienza, Dipartimento SBAI, 00165 Rome, Italy \\ ${ }^{5}$ ELI-NP, IFIN-HH, 077125 Bucharest-Magurele, Romania \\ ${ }^{a)}$ Corresponding author: negoita@nipne.ro
}

\begin{abstract}
The measurement of energy spectra of neutrons and gamma rays emitted by nuclei, together with charge particles spectroscopy, are the main tools for understanding nuclear phenomena occurring also in high power laser driven experiments. However, the large number of particles emitted in a very short time, in particular the strong X-rays flash produced in laser-target interaction, impose adaptation of technique currently used in nuclear physics experiment at accelerator based facilities. These aspects are discussed (Section 1) in the context of proposed studies at high power laser system of ELI-NP. Preliminary results from two experiments performed at Titan (LLNL) and ELFIE (LULI) facilities using plastic scintillators for neutron detection (Section 2) and $\mathrm{LaBr}_{3}(\mathrm{Ce}$ ) scintillators for gamma detection (Section 3) are presented demonstrating the capabilities and the limitations of the employed methods. Possible improvements of these spectroscopic methods and their proposed implementation at ELI-NP will be discussed as well in the last section.
\end{abstract}

\section{NUCLEAR PHYSICS EXPERIMENTS PROPOSED AT ELI-NP HIGH POWER LASER SYSTEM}

It is well known that high power laser pulses can accelerate electrons, protons and heavy-ions to high energies, enough to produce almost all known types of nuclear reaction. At ELI-NP, the reactions induced by accelerated high density ion bunches with secondary targets (solid, gaseous or a laser generated plasma), will be extensively studied focused on specific phenomena that cannot be easily realized at accelerator based nuclear facilities. At intensities approaching $10^{23} \mathrm{~W} / \mathrm{cm}^{2}$, and beyond, that will be achievable at ELI-NP, the dominance of RPA (Radiation Pressure Acceleration) mechanism for ion acceleration over TNSA (Target Normal Sheath Acceleration) is expected [1]. Especially in the "light sail" regime of RPA, quasi-neutral quasi-monoenergetic bunches with solid state density are predicted to be produced, that is about $10^{14}$ higher ion densities compared to existing the ion beam accelerators. High density of reaction opens up the possibility for interaction between unstable reactions products, which is exploited in the proposed fission-fusion scenario [2] to access the region of neutron rich $\mathrm{N} \sim 126$ nuclei. This region of nuclear chart represents a waiting point in astrophysical r-process and therefore is very important in understanding the nucleosynthesis of heavy elements. Before implementing complex and expensive devices such as

Exotic Nuclei and Nuclear/Particle Astrophysics (V). From Nuclei to Stars AIP Conf. Proc. 1645, 228-236 (2015); doi: 10.1063/1.4909579

(C) 2015 AIP Publishing LLC 978-0-7354-1284-2/\$30.00 
magnetic separators and ions traps to select the nuclei of interest produced, the identification and yield measurements has be based on gamma spectroscopy following $\beta$-decay. Activation set-ups have been already used in many laser driven experiments, mainly for characterization of proton and neutron yields. Among them, NATALIE system developed at CENBG[3] is exploiting $\beta^{+}$-decay for $511 \mathrm{keV}-511 \mathrm{keV}$ coincidences measurement reducing the background and achieving low activity measurements. However at ELI-NP, the repetition rates of about 1 shot/minute are expected for $10 \mathrm{PW}$ laser pulses and even higher for $1 \mathrm{PW}$ outputs. Handling of activation samples at such rates is impossible. Therefore a fast transport system is proposed to collect the ions near the production and move them to a decay station where several Ge detectors in close geometry should be installed. The huge X-ray flash and the electromagnetic pulse (EMP) associated to laser-target interaction impose to install such decay station in a very well shielded environment placed at some $10 \mathrm{~m}$ from target.

Another type of nuclear physics experiments proposed at ELI-NP is related to nuclear excitations and deexcitation in (hot) plasma. Various mechanisms may appear in nuclear transitions due to modifications in the electron shell or due to interactions with free electrons/X-rays/ $\gamma$-rays. In the first category we include:

- Internal Conversion (IC): the well-known nuclear de-excitation mechanism with emission of conversion electrons

- Bound Internal conversion (BIC): Same as IC but electron is promoted to a bound state.

- NEEC (Nuclear excitation following electron capture) - inverse of IC.

- NEET (Nuclear Excitations following an electronic transition) - inverse of BIC

Significant changes in nuclear lifetimes are predicted[4] for isomeric states and not only. For example,one of the most interesting constraints ofthe astrophysical models comes from the gamma ray mapping of ${ }^{26} \mathrm{Al}$ across the Galaxy. This radio-isotope is produced through Asymptotic Giant Branch (AGB) and Wolf Rayet stars. Theoretical work [5] based on shell-model calculations predicts a dramatic reduction of the effective life time $\tau_{\text {eff }}{ }^{26 g s} \mathrm{Al}$ ) by a factor of $10^{9}$ within the temperature range from 0.15 to $0.4 \mathrm{GK}$. Laser of moderate intensities can indeed produce hot plasma at various densities resembling astrophysical environments. Expansion of such plasma is very rapid. Therefore the best way to produce the nuclear states to be studied is to use laser accelerated ion bunches having precise timing and densities much higher than conventional accelerators. Activation method mentioned above can be employed also for such studies, provided that nuclear lifetime are enough large to allow for transportation. However, the development of in-situ gamma spectroscopy may allow measurement of very short lifetime and enlarge the number of cases that can be studied. In the Section 3 we present the first successful attempts to develop in-situ gamma spectroscopy using the new type of fast, high output, $\mathrm{LaBr}_{3}$ :Ce scintillators.

The couplings between electron shells and nuclei affect also the nuclear reactions, not only the nuclear structure and lifetimes. The screening effect is accounting for difference between cross section of bare nuclei (measured with indirect methods) and cross section measured at low energies were electrons partly screen the Coulomb repulsion the two interacting nuclei. This effect, responsible for significant enhancement of cross section, is dependent on target conditions. Its dependence on plasma temperatures and densities is very importance for astrophysics since reaction rates, energy production, composition evolution, etc are directly related to cross sections. One of the experimental studies proposed at ELI-NP is aiming to measure the cross section of few astrophysical relevant reactions using laser accelerated ions impinging a laser created plasma. Variation of gas density and of the parameters of the laser used for plasma creating allows studying the cross section modification and gaining a better understanding of screening effect. The activation method could provide at best an yield integrated over all the energies involved, while measurement of produced neutron spectrum offers the possibility to extract the reaction rates as function of reaction energy. The measurement of neutron spectra is general a rather difficult task. In laser driven experiments, in order to cope with X-rays flash, bubble detectors has been employed as they are insensitive to gamma and X-ray. Their energy resolution and efficiency are, nevertheless, insufficient in most cases. Gas $\left({ }^{3} \mathrm{He}\right)$ detectors have, qualitatively speaking, the same advantages and disadvantages. Instead, the use of plastic scintillators and time-of-flight techniques has been suggested, since their efficiency is much higher. However, large amount of lead shielding are needed to avoid saturation of scintillator and photomultiplier tube (PMT) such that to see the signal induced by neutrons arriving to detectors just few tens or hundreds of nanoseconds after the prompt $\mathrm{X}$ and gamma rays. This method has been applied for example in [6]. The goal of this experiment was to develop a brilliant fast neutron source for applications. We applied a similar method in a recent experiment at Titan facility at LLNL (SUA), the aim was to produce a short duration quasi-monoenergetic neutron source as probe for fast, transient phenomena. This experiment and some preliminary results of it are presented in the next section. 


\section{NEUTRON TIME-OF-FLIGHT SPECTROSCOPY IN LASER DRIVEN EXPERIMENTS}

Experiment described was performed at Titan short-pulse laser of LLNL proving laser pulses with up to $250 \mathrm{~J}$ compressed down to $600 \mathrm{fs}$, that is $1 \mathrm{PW}$-class laser. The experiment aimed to develop a short duration neutron source using (i) laser proton acceleration from a thin primary target, (ii) proton energy selection using the method from [7] and (iii) reaction of protons in a secondary LiF target. In order to measure the neutron production a number of 11 plastic scintillator detectors available in IFIN-HH have been placed at various angles and distances, shielded by $30 \mathrm{~cm}$ of lead in the direction of target and at least $2.5 \mathrm{~cm}$ in the other directions (see Figure 1). Dimensions of scintillator are $4 \times 4 \times 12 \mathrm{~cm}^{3}$ and they were coupled to 1.5 " diameter photomultipliers. The detector signals have been readout by a 32 channel 1 GSamples/second digitizer trigger by the laser pulse.

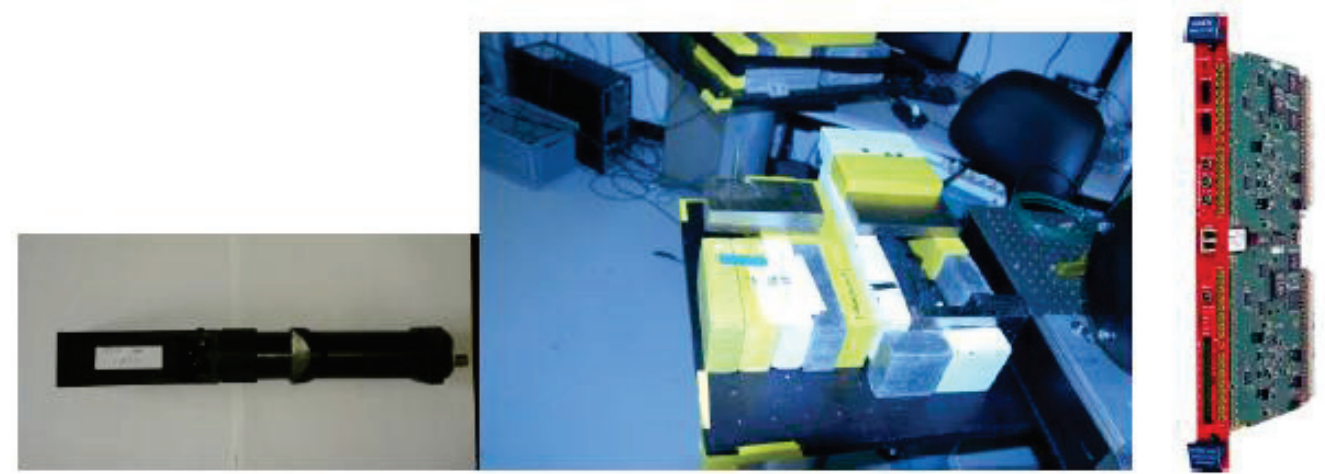

FIGURE 1. The scintillator detector outside (left photography) and inside lead shielding (middle), and the digitizer (right) employed for signal acquisition.

In Figure 2 the traces for one detector and three laser shots are plotted. In nuclear physics experiments, the neutron time-of-flight spectra are usually obtained for a series of individual neutron interactions with detector, each generating a signal that is passed through a discriminator (in most of the cases a constant fraction discriminator) and giving a count in a channel of ToF histogram. This is not the case for the spectra in figure 2 . They are the result of the overlap of signals from a large number of neutrons that hit de detector almost in the same time. Therefore, the determination of incident neutron spectrum from measured signal requires a fitting procedure that has to take into account explicitly the distribution of signal shapes and amplitudes associated to incident neutrons, distribution that is function of neutron energy and incidence geometry on the detector. Consequently, the data analysis method has to be based on extensive simulations with adjustments of parameters (detector size and material compositions, photomultiplier amplification and signal width, neutron and gamma interactions included in simulation) to reproduce experimental data obtained with known neutron flux.

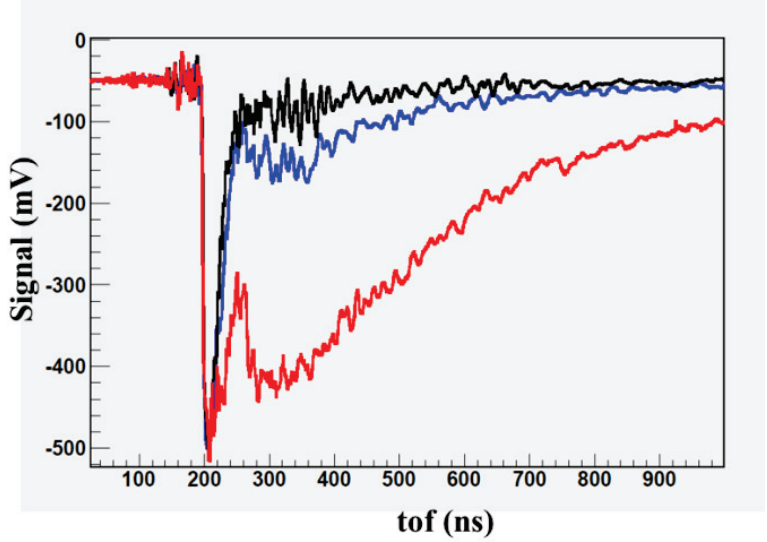

FIGURE 2. Experimental data obtained from one detector with full energy spectrum of accelerated protons on the LiF target (red signal), with energy selected protons on the same LiF target (blue signal) and without LiF target (black signal). 
The large quantity of lead shielding is an additional complication that needs careful comparison of simulation and experimental data. Despite the fact that experimental data taken in such conditions are reported in the literature, the proposed consistent approach described above for data analysis has been not yet applied. The performed simulation used the GEANT4 toolkit [8] developed at CERN turning on all processes relevant to neutron interactions and secondary particles (protons, alpha, gamma, electrons). The scintillation photon yields as function of particle type and deposited energy were implemented according to V.V.Verbinski et al.[9], but the propagation of these optical photons has been not simulated. For each incident event, the full history of interactions with sensitive volume of the detector has been saved and analyzed using the root framework [http://root.cern.ch]. Thus, up to a constant, the distribution of scintillation photons on the photocathode is obtained and then convoluted to the response function of the photomultiplier (as filtered by the digitizers and measured with gamma radiation sources). Finally, the signal corresponding to a laser shot is obtained as sum of signals due to many individual neutrons generated isotropic at target position according to a given initial energy distribution. The results of such simulation are presented in Figure 3 for a flight path of $2 \mathrm{~m}$. One can observe the reduction in signal amplitude by a factor of 5 when $20 \mathrm{~cm}$ of lead is added just in front of detector without significant distortion of signal shape. However, when the lead is installed at about $1 \mathrm{~m}$ in front of detector the distortion becomes significant even for $10 \mathrm{~cm}$ of lead. The condition put on the rather narrow range of initial neutron energies (red curves in Figure 3) demonstrates that lead do not increase the time of flight, that is many neutrons emitted toward detector and eventually scattered at small angles hit the detectors while the multiple, large angle, scattered neutrons are no longer inducing significant signal. No spurious increase at large times is observed. Beside the detectors and their lead shielding, the Al interaction target (having $10 \mathrm{~cm}$ thickness) as well as concrete walls of the experimental hall has been included in the simulation leading however to the conclusion that their influence on signal shape is very small.
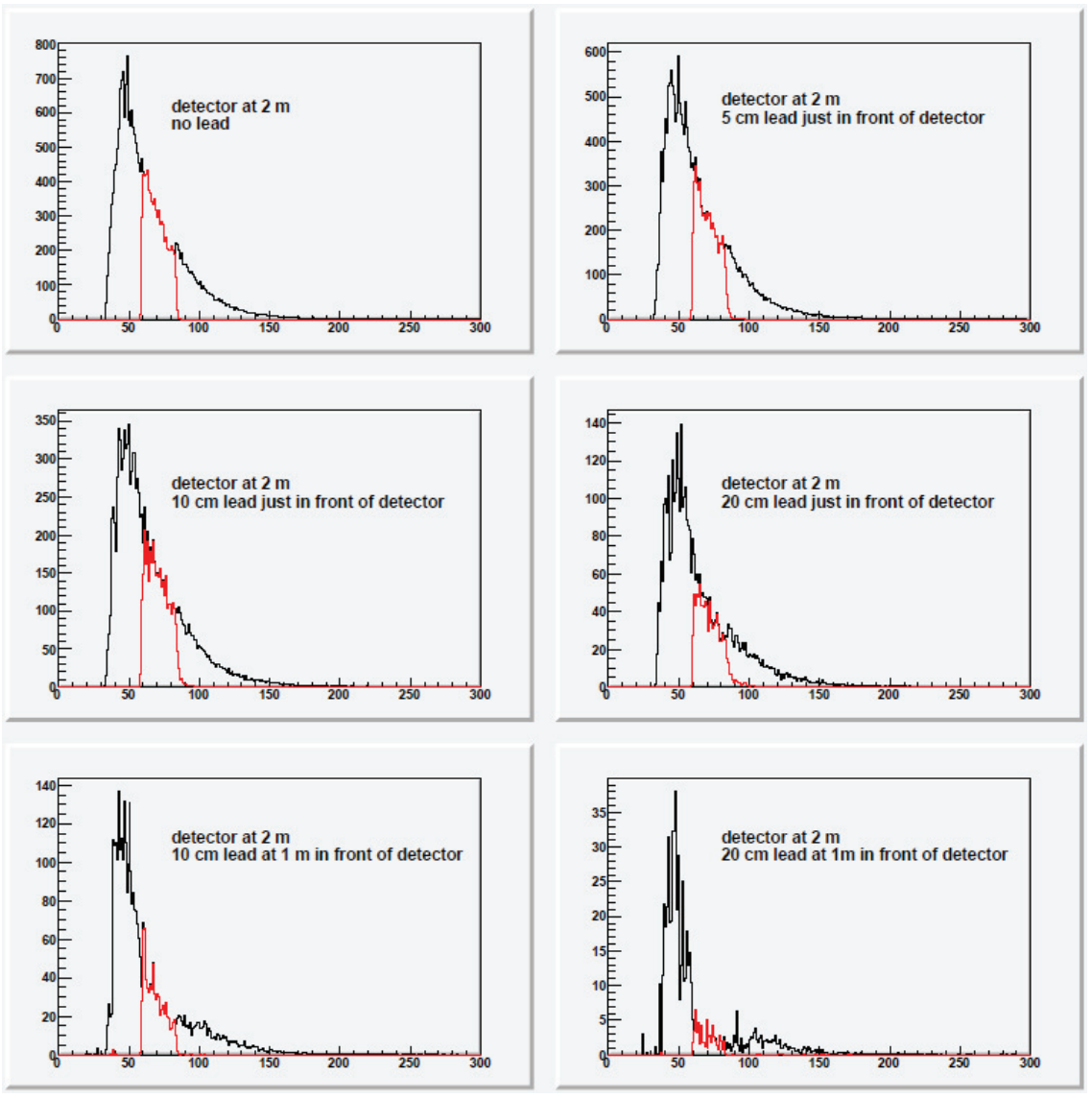

FIGURE 3. Simulated signal waveforms (photon number in a. u. versus time in ns) for a flat distribution of initial neutron energies between $0.1 \mathrm{MeV}$ and $20 \mathrm{MeV}$ in various configurations of the shielding, as mentioned on each graph. The convolution with detector response function to single scintillation event is not included for these waveforms. The red line corresponds to neutrons restricted to $3-6 \mathrm{MeV}$ initial energy range. 


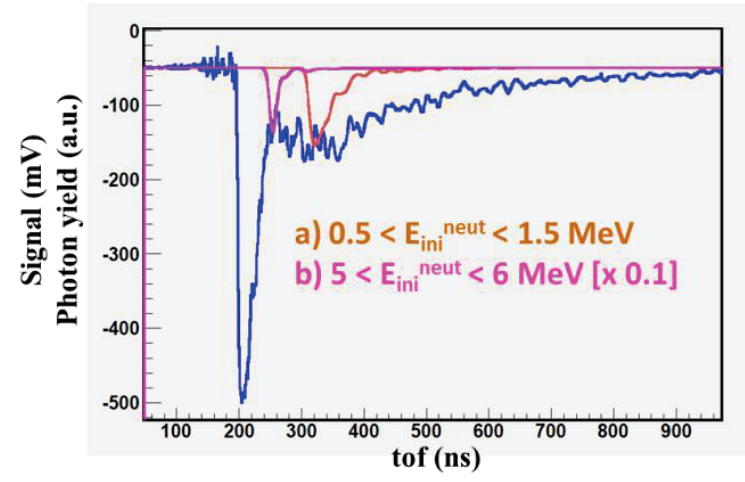

FIGURE 4. Simulations of photon yield in the scintillator for neutrons with initial energy in the mentioned ranges compared with the experimental signal plotted with same color as in Figure 2.

The above conclusion based on simulation have been check recently in an experiment at IFIN-HH Tandem. It proves that the method works for lead shielding up to about $30 \mathrm{~cm}$. Further, in order to extract from measured signals the energy distribution of the generated neutrons a deconvolution/fitting procedure has to be applied. It is shown qualitatively in the Figure 4: calculated signals for two narrow energy intervals (1 MeV) are superimposed on the measured experimental waveform. The signals were convoluted with a Gaussian of $10 \mathrm{~ns}$ which is the measured averaged width of the signal of the detector exposed to a gamma source. We mention also that this detector has been shielded by $30 \mathrm{~cm}$ of lead and the same thickness has been used in simulations presented in this figure. Compared to simulated (calculated) signals in Figure 3, we observe a more produced enlargement toward large times meaning that $30 \mathrm{~cm}$ is an upper limit for shielding in case of neutron energies below $1 \mathrm{MeV}$.

It is important to note that the signal corresponding to neutrons in the $5-6 \mathrm{MeV}$ range (magenta signal) has be reduced by a factor of 10 in order to obtained a height of the signal similar to the one corresponding to neutrons in 0.5-1.5 MeV range (brown signal) for the same number of incident neutrons. This means that the low energy neutrons (below $1 \mathrm{MeV}$ ) have an important contribution in the generated spectrum.

\section{IN-SITU GAMMA SPECTROSCOPY IN LASER DRIVEN EXPERIMENTS}

In this section we report the preliminary results from an experiment at ELFIE, the 100 TW facility at LULI demonstrating the feasibility of in-situ measurement of short-lived nuclear isomers. In Figure 5 the position of the detector inside interaction chamber and the target-activation foil assembly is shown.
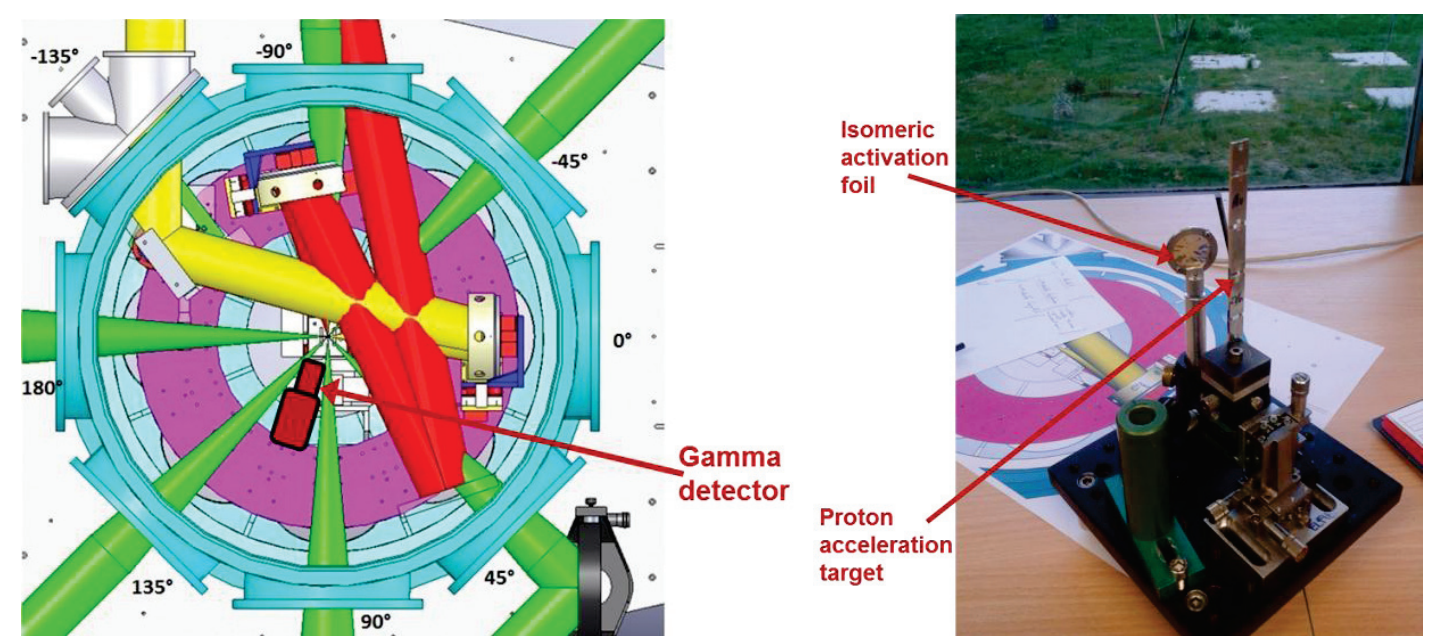

FIGURE 5. Left: configuration of the laser beams available at ELFIE facility. Only the yellow beam (20 J - $350 \mathrm{fs})$ has been used in described experiment. Position of gamma detector at about $10 \mathrm{~cm}$ from target is also shown. Right: The target for proton acceleration placed in the laser focus and the isomeric activation foil (secondary target) installed at about $4 \mathrm{~cm}$ downstream. 
As a first successful attempt to use gamma detectors in-situ we have to mention the work of R. J. Clark et al. [10]. The $511 \mathrm{keV}$ gamma rays following the annihilation of positrons emitted in $\beta^{+}$-decay of ${ }^{27} \mathrm{Si}$ have measured with a $\mathrm{NaI}$ detector. The lifetime of ${ }^{27} \mathrm{Si}$ isotope is $4.2 \mathrm{~s}$ and it has been produced by a $(\mathrm{p}, \mathrm{n})$ reaction on a $\mathrm{Al}$ target. The saturation of the detector due to gamma flash lasted about 1 second. A second component in the decay curve has been identified and attributed to ${ }^{26 \mathrm{~m}} \mathrm{Al}$ isomer with a known lifetime of $6.3 \mathrm{~s}$. Since reaction threshold for production of the isomer is much higher compared to ${ }^{27} \mathrm{Si}$, one can deduce the slope of the exponentially proton spectrum from the measurement of the yield of the two states.Unfortunately the isomer decay proceeds $100 \%$ though $\beta+$ decay and, similar to decay of ${ }^{27} \mathrm{Si}$, no gamma ray is emitted from daughter nuclei such that separation of the two states is not possible, such that theproduction of each state can obtained only throughan accurate fit of the decay curve corresponding to $511 \mathrm{keV}$ line.

In our work at LULI we used a nat $\mathrm{Zr}$ foil (having $51 \%$ abundance of ${ }^{90} \mathrm{Zr}$ ) which may produce several isomeric states emitting specific gamma rays: $2319 \mathrm{keV}$ with $809 \mathrm{~ms}$ lifetime in ${ }^{90} \mathrm{Zr}\left(\mathrm{p}, \mathrm{p}^{\prime}\right)^{90 \mathrm{~m}} \mathrm{Zr}$ reaction, and $257 \mathrm{keV}-$ $6.2 \mathrm{~ms}, 124 \mathrm{keV}-18.8 \mathrm{~s}$ and $122 \mathrm{keV}-63 \mu \mathrm{s}$ in ${ }^{90} \mathrm{Zr}(\mathrm{p}, \mathrm{n}){ }^{90 \mathrm{~m}} \mathrm{Nb}$ reaction. For gamma ray detection we used an $\mathrm{LaBr}_{3}$ :Ce crystal of 1.5 " diameter and length, coupled to a gated photomultiplier. The scintillation yield of this new material is $160 \%$ relative to NaI giving accordingly better energy resolution, while scintillation decay constant of $16 \mathrm{~ns}$, compared to $250 \mathrm{~ns}$ of NaI, imposed the $\mathrm{LaBr}_{3}$ detectors as first choice in gamma timing measurement in nuclear physics. The signal from our detector was split in two. One branch was sent to an oscilloscope for digitization. The second branch to a fast amplifier for pulse stretching and then fed into TNT2 [11] card equipped with $100 \mathrm{MS} / \mathrm{s}$ and on board pulse shape analysis proving on-line pulse amplitude and arrival time.
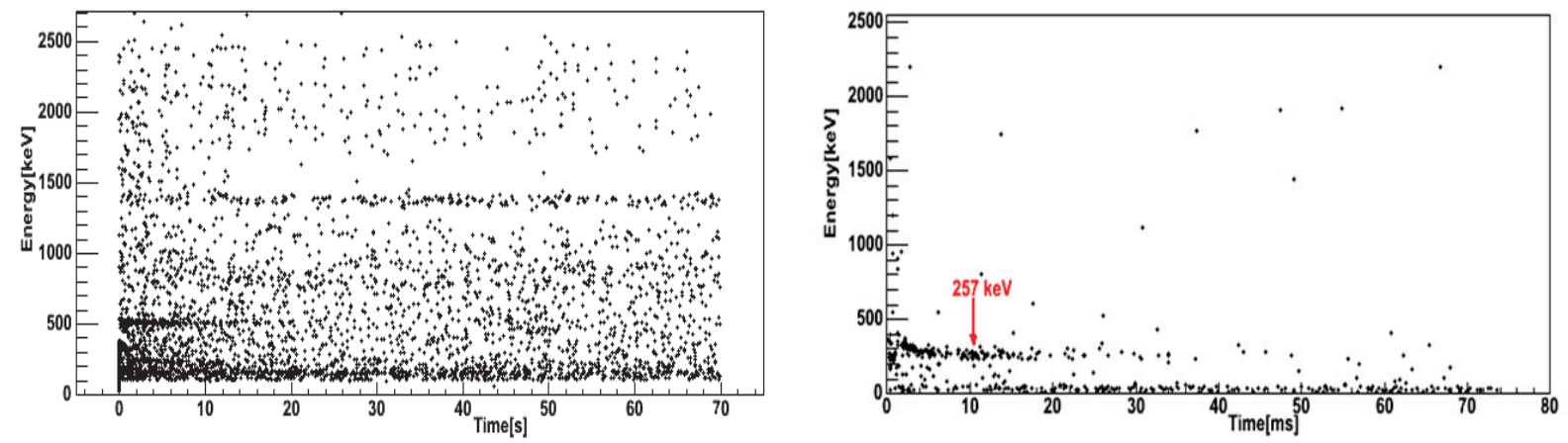

FIGURE 6. Bidimensional plots of gamma events recorder following one (single) laser shot. Time is measured relative to laser shot arrival.

Off-line analysis of traces recorded by the oscilloscope produced the same results as TNT, which are show in Figure 6. In the graph on the left one can observe the background line of $1436 \mathrm{keV}$ (internal contamination of the $\mathrm{LaBr}_{3}(\mathrm{Ce})$ crystal), the $511 \mathrm{keV}$ line corresponding most probably to reactions on aluminum holder of the foil, a weaker line at $208 \mathrm{keV}$ identified as $5.1 \mathrm{~s}$ isomer in ${ }^{79} \mathrm{Br}$ populated in $\left(\gamma, \gamma^{\prime}\right)$ photonuclear reaction inside detector itself and, on the bottom side just above the low energy background, the $122 \mathrm{keV}$ line extending to tens of seconds. This last observation is explained by level scheme of the decay of the $124 \mathrm{keV}$ state which feds the $121 \mathrm{keV}$ by a low energy transitions. On the right graph in Figure 6 a zoom on time scale is shown. The $257 \mathrm{keV}$ transition with characteristic decay time of $6 \mathrm{~ms}$ is well see, on a very low background. We note that more the 100 counts have been measured for each isomeric transition. Taking into account the efficiency of gamma detection we conclude that more than $10^{6}$ isomeric states are produced per shot. A more quantitative comparison of experimental number of counts with the expected values calculated from the measured proton spectrum (using radiochromic films) folded with cross section computed with TALYS code [12] reveals a factor of about 2 . This is actually a reasonable factor taking into account, on one hand, that cross section for population of isomeric state implies interplay of nuclear structure and reaction mechanism difficult to be described by theoretical models and, on the other hand, uncertainties of the procedure of determination of proton spectrum using the radiochromic films.

The plots in Figure 6 clearly demonstrate the feasibility of performing gamma spectroscopy of short live nuclear isomers with lifetimes down to millisecond. Our attempts to achieve even shorter lifetimes were not successful. The reduction of saturation and recovery time observed in Figure 6 has been tried varying the gate of the photomultiplier and by shielding the detector with about $2 \mathrm{~cm}$ of lead all around, except in the direction of $\mathrm{Zr}$ foil. No significant improvements have been observed. Further possible improvements are discussed in the next section. 


\section{PERSPECTIVES AT ELI-NP}

In this section, we are referring to laser driven nuclear physics experiments at ELI-NP as studies using only the High Power Laser System (HPLS) and not involving the Gamma Beam System (GBS). Production of gamma beam through invers Compton process involves also a rather high power short pulse laser (of about $1 \mathrm{TW}$ ) but it is not part of HPLS. Laser driven nuclear physics experiments are planned mainly in E1 area where the two 10 PW pulses will be available. Additionally, the two 1 PW outputs of HPLS as well as the two $0.1 \mathrm{PW}$ outputs available in the E5 area, and respectively E4 area, could also be used for nuclear physics experiments. The interest is related to higher repetition rates of these intermediate outputs of HPLS: 1 pulse per minute for $10 \mathrm{PW}$ compared to $1 \mathrm{~Hz}$ for $1 \mathrm{PW}$ and $10 \mathrm{~Hz}$ for $0.1 \mathrm{PW}$ which could increase in some cases the average yields for the nuclear states or reactions of interest. Such repetition rates at 1 PW and 100 TW power levels are already available in the world. However, ELINP will offer to the users instrumentation specific to laser driven nuclear physics experiments that will be hardly available to other facilities. The proposed equipment will be described further below, after a brief presentation of laser configurations in E1 area.

Three main configurations have been identified as shown in Figure 7:

(a) Perpendicular directions of focusing configuration means that one $10 \mathrm{PW}$ beam is used for ion acceleration tightly focused on solid target while the second $10 \mathrm{PW}$ beam, that can also a long duration(uncompressed) pulse of about $300 \mathrm{~J}$ and $1 \mathrm{~ns}$, will be used for gas plasma heating, or microlenses charging, or a high $\mathrm{B}$-field production. The distance between the two foci can be varied as well as the timing. The acceleration beam will be available with circular polarization.

(b) The small angle focusing configuration is meant to provide maxim possible intensity on target combining the two $10 \mathrm{PW}$ pulses. Circular polarization for both beams is requested.

(c) The configuration with a plasma mirror before target will be probably the first to be implemented in order to diminish the pollution of off-axis parabola with target debris. The problem of target debris mitigation and definition of efficient cleaning procedures for large optics is very important for operational phase, because of large number of $10 \mathrm{PW}$ pulses requested to be delivered (few hundreds per day) and of the large cost and long duration of mirror (re)polishing. A second laser pulse, of $1 \mathrm{PW}$ beam (or $30 \mathrm{~J}$ with about 1 ns duration) as shown in figure 7(c), is proposed to be added in this configuration too.

These configurations, defined mainly by the focal length and deflection angles of off-axis parabolas, has been chosen taking into account the needs of experiments in other areas, trying to minimize the number of different parabolas and allowing interchange of components between experimental set-ups.

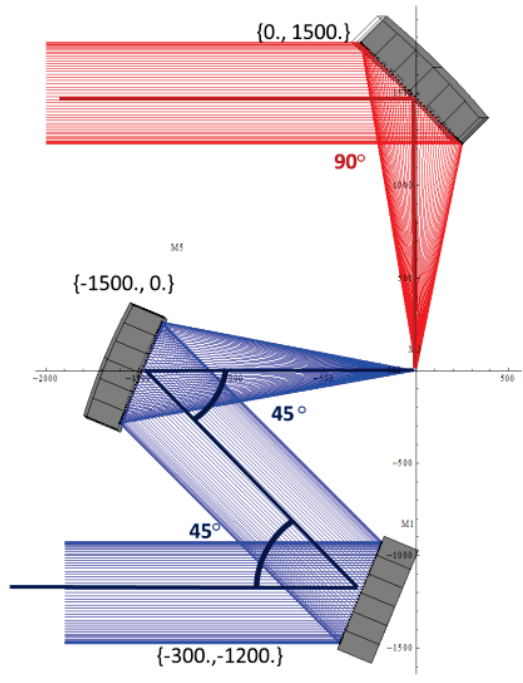

(a)

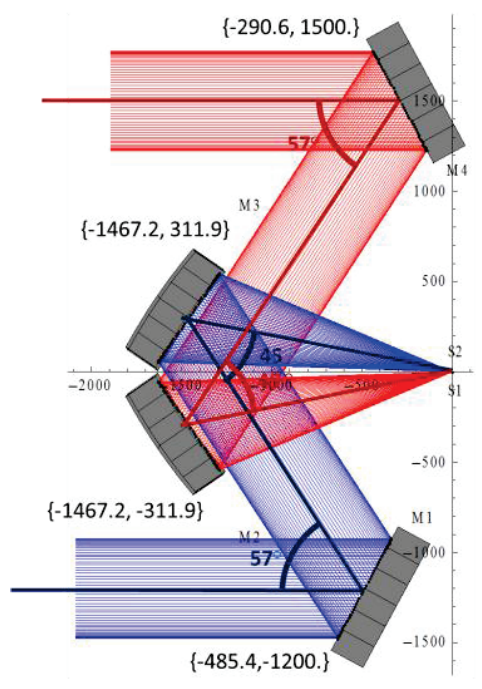

(b)

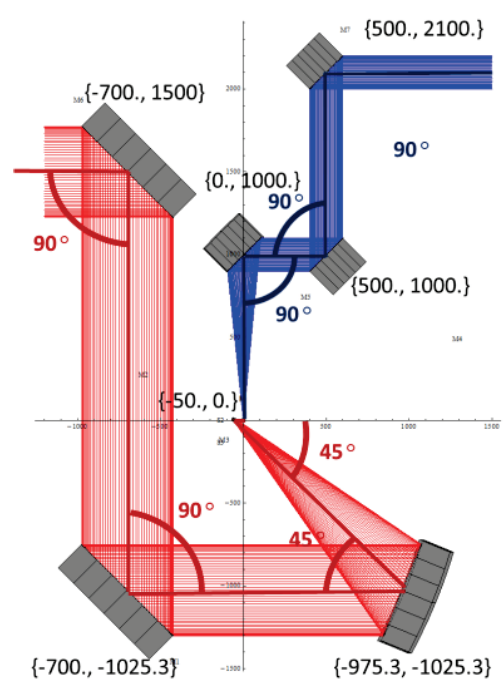

(c)

FIGURE 7. Lasers beam configurations proposed for experiments in E1 area (see text for details) shown in a top view and target position in the origin of the axis. Axis units are $\mathrm{mm}$. The diameter of $10 \mathrm{PW}$ beams is $500 \mathrm{~mm}$ and focal length of corresponding off-axis parabola is $1500 \mathrm{~mm}$. Dark blue beam in figure (c) represents a $1 \mathrm{PW}$ laser beam. 


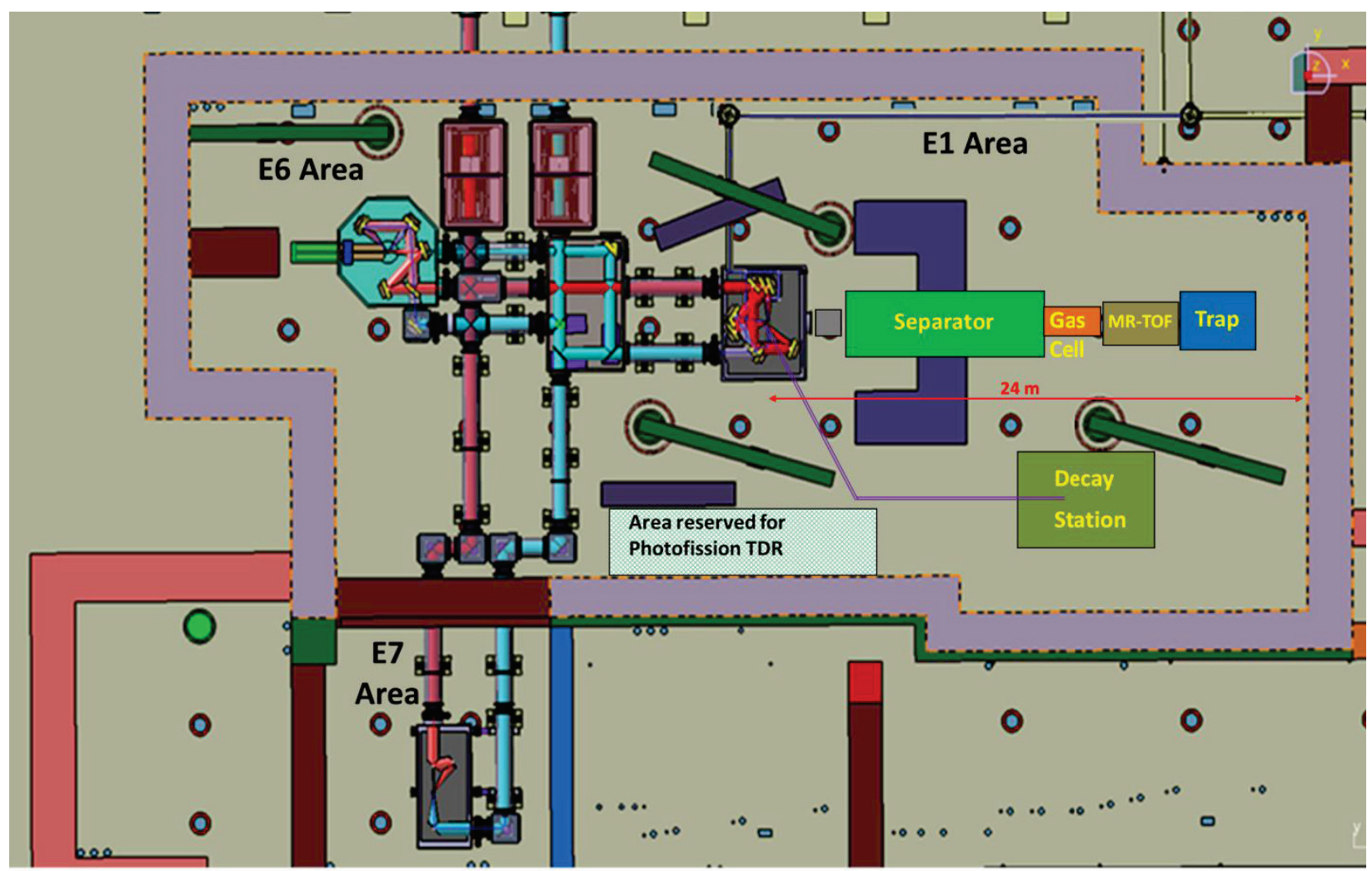

FIGURE 8. Layout of experimental areas where the two $10 \mathrm{PW}$ beams are distributed.

The layout of E1 area is shown in Figure 8. The configurations presented in Figure 7 are superimposed inside the central rectangle representing the interaction chamber with dimensions of more than $3 \times 4 \mathrm{~m}^{2}$. Along acceleration direction a $\mathrm{C}$ shape beam-dump will be installed and several instruments are envisaged: an electromagnetic separator is proposed for in-flight selection of fission-fusion reaction products followed by a stopping gas cell and devices for accurate mass measurements. This complex equipment will be optimally designed after the demonstration and characterization of heavy ion acceleration through RPA mechanism at $\sim 10^{23} \mathrm{~W} / \mathrm{cm}^{2}$ intensity level and installed in later phase of development.

In the first phase, a decay station will be built and a fast transport system will bring the radioactive reaction products in front a few Ge detectors for identification and yield measurement through high resolution gamma spectroscopy. A tape system or aerosols in fast gas flow are taken into consideration for the transport system. This method can collect only production with enough energy to reach the moving element of the transport system. For the remaining radioactive elements in the target or isomers, the known methods of activation measurement for particles characterization (complemented by autoradiography using image plates) will be available. However, the in-situ gamma spectroscopy techniques mentioned in section 3 will take true advantage of high repetition rate of HPLS. The ongoing $\mathrm{R} \& \mathrm{D}$ to improve this technique is focused on lead shielding optimization and addition to detector assembly a fast LED able to emitted short light pulses that will allow us to apply a correction to the signal amplitude during the recovery period. The perspective of measuring gamma decay of states with lifetimes down to 10 microseconds seems feasible. The interaction chamber is enough large to accommodate several detectors, possible hanging to an angle and pointing to a secondary target or catcher, even if the large and heavy lead shielding will be needed. On line processing has to be also developed in order to get full benefits from the system: one-dimensional and bi-dimensional spectra, corrected by amplitude and calibrated in energy and time, has to be built for each shot as well as for sequence of shots.

Regarding neutron spectroscopy we remark that large flight paths are assured around interaction chamber: at least $4 \mathrm{~m}$ in all directions and much more at some particular emission angles. Determination of energy distribution with discrimination of single hits is obviously preferred to the method of fitting a signal corresponding to overlapping multi-neutron described in section 2 . However it means that a large number of detectors are required to 
obtain relevant statistics for a single shot. An array of hundreds of small plastic scintillators coupled to silicon photomultipliers (SPM) is currently under consideration [13] as cheaper and more compact alternative to dynode based photomultipliers tubeswhich could significantly reduce also the total amount lead shielding. However, while EMP is only slightly disturbing the signal of conventional PMTs, in case of SPM consistent EMP shielding might be required. The large number of digitizers is needed and performs off-line discrimination and charge integration without signal splitting with adequate signal treatment.

In conclusion, the neutron and gamma spectroscopy techniques has to be deeply reconsidered and adapted to experimental conditions encountered in the studies using high power laser system. Implemented at ELI-NP they will offer to users unique opportunities for laser driven nuclear studies on top of other state-of-the-art equipment specific to lasers research infrastructures.

\section{ACKNOWLEDGMENTS}

We acknowledge the support of the European Commission, under the project CRISP, FP7 contract number 283745 and of Romanian National Authority for Scientific Research, CNCS/UEFISCDI, under the project number PN-II-ID-JRP-2011-1.

The ELI-NP project is co-funded by The European Union through the European Regional Development Fund.

\section{REFERENCES}

1. A.P.L. Robinson et al., New J. Phys. 10 (2008) 013021

2. D. Habs et al., Appl. Phys. B 103 (2011) 471

3. M.Tarisien et al., Rev.Sci.Instr. 82 (2011) 023302

4. G. Gosselin et al; Phys. Rev. C 70 (2004) 064603

5. A. Coc et al.., Phys. Rev. C 61 (1999) 015801

6. M. Roth et al., Phys. Rev. Lett.110 (2013), 044802

7. T. Toncian, et al., Science 312 (2006) 410

8. S. Agostinelli et al.,Nucl. Instrum. Meth.A 506 (2003) 250

9. V.V.Verbinski et al., Nucl. Instrum. Meth. 65 (1968) 8

10. R. J. Clarke et al., Appl. Phys. Lett. 89 (2006) 141117

11. http://www.iphc.cnrs.fr/-TNT-.html

12. http://www.talys.eu

13. J.Y Yeom et al., IEEE Phot. Tech. Lett. 25 (2013) 1309 